November 26, 2018 13:8 WSPC/INSTRUCTION FILE ParisBrownUproc

\title{
GRAVITATIONAL INSTABILITY OF THE VACUUM AND THE COSMOLOGICAL CONSTANT PROBLEM
}

\author{
J. W. MOFFAT \\ Perimeter Institute for Theoretical Physics, Address \\ 31 Caroline St. North, Waterloo, Ontario, Canada \\ Department of Physics, University of Waterloo, Address \\ Waterloo, Ontario, Canada
}

\begin{abstract}
A mechanism for suppressing the cosmological constant is described, using a superconducting analogy in which fermions coupled perturbatively to gravitons are in an unstable false vacuum. The coupling of the fermions to gravitons and a screened attractive interaction among pairs of fermions generates fermion condensates with zero momentum and a phase transition induces a non-perturbative transition to a true vacuum state. This produces a positive energy gap $\Delta$ in the vacuum energy identified with $\sqrt{\Lambda}$, where $\Lambda$ is the cosmological constant. In the strong coupling limit, a large cosmological constant induces a period of inflation in the early universe, followed by a weak coupling limit in which $\sqrt{\Lambda}$ vanishes exponentially fast as the universe expands due to the dependence of the energy gap on the density of Fermi surface fermions, predicting a small cosmological constant in the early universe.
\end{abstract}

\section{Cosmological Constant Problem, Vacuum Energy and Inflation}

It is generally agreed that the cosmological constant problem (CCP) is one of the most severe problems facing modern particle and gravitational physics. It is believed that its solution could significantly alter our understanding of particle physics and cosmology. The problem has become more severe with the discovery that the universe is accelerating and that the so-called "dark energy" can be explained by a non-zero and small cosmological constant.

The cosmological constant problem centers around two questions: why is the cosmological constant observed to be so small and why does it dominate the universe today 12. Many investigators have proposed solutions ranging from a rolling scalar field to the anthropic principle. However, it can be argued that the greatest irony of the cosmological constant problem is the inflationary paradigm. The onset of the inflationary epoch is completely dominated with a fluid that is either a pure cosmological constant or a scalar field whose potential is extremely flat, mimicking a cosmological constant. Clearly this period of inflation has to end for successful structure formation. However, whatever mechanism is responsible for this exit from inflation should be a clue as to how the cosmological constant is relaxed at all times including today. 
We shall propose a mechanism to solve the cosmological constant problem, based on a simple idea analogous to the microscopic realization of superconductivity $\underline{3}$. We argue that the perturbative vacuum state in the presence of a cosmological constant is gravitationally unstable and it is energetically favorable for the vacuum associated with the effective cosmological constant to release all of its energy into the production of fermion condensates. The formation of condensates leads to a non-perturbative true ground state. The non-perturbative gravitational instability of the vacuum due to a transition of fermions to a superfluid of condensates in the early universe, produces a large cosmological constant which induces a de Sitter expansion and inflation, followed by an exponential suppression of the cosmological constant as the universe expands.

We can define an effective cosmological constant

$$
\lambda_{\text {eff }}=\lambda_{0}+\lambda_{\mathrm{vac}}
$$

where $\lambda_{0}$ is the "bare" cosmological constant in Einstein's classical field equations, and $\lambda_{\text {vac }}$ is the contribution that arises from the vacuum density $\lambda_{\mathrm{vac}}=8 \pi G \rho_{\mathrm{vac}}$.

Already at the standard model electroweak scale $\sim 10^{2} \mathrm{GeV}$, a calculation of the vacuum density $\rho_{\mathrm{vac}}$, based on local perturbative quantum field theory results in a discrepancy of order $10^{55}$ with the observational bound

$$
\rho_{\mathrm{vac}} \leq 10^{-47}(\mathrm{GeV})^{4} \sim\left(10^{-3} \mathrm{eV}\right)^{4} .
$$

If the vacuum energy is the dark energy, then $\rho_{\text {vac }} \sim\left(10^{-3} \mathrm{eV}\right)^{4}$. There is an egregious discrepancy between the particle physics estimate of $\rho_{\mathrm{vac}}$ and the cosmological observation. We are faced with a severe fine-tuning problem of order $10^{55}$, since the virtual quantum fluctuations giving rise to $\lambda_{\text {vac }}$ must cancel $\lambda_{0}$ to an unbelievable degree of accuracy. This is the "particle physics" source of the cosmological constant problem.

In order to achieve at least 60 e-folds of inflation, we need to postulate a large vacuum energy initially in the early universe, which generates a de Sitter phase of inflation

$$
a(t) \sim \exp \left(H_{0} t\right) \sim \exp (\sqrt{\Lambda / 3} t) .
$$

A major problem for inflationary models is to explain why the cosmological constant (vacuum energy) is very small in the present universe. The usual statement is that the vacuum energy decays into particles but this implies a severe fine tuning and it is not accompanied by a satisfactory particle physics description of the mechanism that underlies the suppression of the initial large vacuum energy.

We will argue that the perturbative vacuum for fermions interacting with gravitons is unstable to the production of massive condensate states, transmuting the large vacuum energy into rest mass. In the case of inflation, these states are the Goldstone bosons corresponding to the broken de Sitter space-time symmetry which commences with a lower cosmological constant. Let us recall that this situation is similar to the vacuum instability phenomena of superconductivity in condensed 
matter systems and QCD at finite density. In the BCS 4 theory of superconductivity, the naive ground state associated with the Fermi surface is unstable in the presence of an attractive electron-phonon interaction, which acts to screen the repulsive Coulomb interaction. This instability is quantified by a gap equation, which is an energy gap $\Delta$ between the superconducting true ground state and the ordinary conduction state.

\section{Vacuum Instability and Fermion Condensates}

We propose a non-perturbative mechanism to solve the cosmological constant problem, based on an analogy with the microphysical realization of superconductivity We argue that in the absence of gravitational interactions between fermions, $\Lambda=0$ and when the fermions exchange gravitons Minkowski spacetime becomes unstable. A non-perturbative phase transition to a true vacuum state occurs when the gravitational interaction is taken into account. The fermions form condensates with zero momentum due to the weak gravitational interaction and a screened long-range attractive interaction among the pairs of fermions.

We shall describe the phase transition to fermion condensates using a nonrelativistic toy model. The Hamiltonian takes the BCS form with $\mathbf{k}=-\mathbf{k}$ :

$$
H=\sum_{k s} \mathcal{E}_{k} c_{k s}^{\dagger} c_{k s}-\frac{1}{2} \sum_{k, k^{\prime} s} V_{k k^{\prime}} c_{k^{\prime} s}^{\dagger} c_{-k^{\prime} s}^{\dagger} c_{-k^{\prime} s} c_{k s}
$$

We perform the transformation to new operators

$$
b_{k}=u_{k} c_{k}-v_{k} c_{-k}^{\dagger}, \quad b_{-k}=u_{k} c_{-k}+v_{k} c_{k}^{\dagger},
$$

where the bs satisfy anti-commutation relations and $u_{k}^{2}+v_{k}^{2}=1$. The fermion number operators are $n_{k}=b_{k}^{\dagger} b_{k}$ and $n_{-k}=b_{-k}^{\dagger} b_{-k}$.

\section{Gap Equation and the Cosmological Constant}

We must now determine the ground state (vacuum) and set the occupation numbers $n_{k}$ and $n_{-k}$ equal to zero. We need to determine the energy gap $\Delta$ produced by the gap in the vacuum energy in the phase transition to the fermion condensates. The condensates are bound states due to the weak gravitational interaction generated by the exchange of gravitons between fermions and the screened attractive force. This will give

$$
\Delta=\sqrt{\Lambda} .
$$

To find the minimum of the BCS energy, we diagonalize $H$ and obtain the condition

$$
\mathcal{E}_{k}\left(\frac{1}{4}-x_{k}^{2}\right)^{1 / 2}+x_{k} \sum_{k^{\prime}} V_{k k^{\prime}}\left(\frac{1}{4}-x_{k^{\prime}}\right)^{1 / 2}=0,
$$

where $u_{k}=\left(\frac{1}{2}-x_{k}\right)^{1 / 2}$ and $v_{k}=\left(\frac{1}{2}+x_{k}\right)^{1 / 2}$. 
4 J. W. Moffat

Let us define the quantity

$$
\Delta_{k}=\sum_{k^{\prime}} V_{k k^{\prime}}\left(\frac{1}{4}-x_{k^{\prime}}\right)^{1 / 2}
$$

giving

$$
\Delta_{k}=\frac{1}{2} \sum_{k^{\prime}} V_{k k^{\prime}} \frac{\Delta_{k^{\prime}}}{\left(\mathcal{E}_{k^{\prime}}^{2}+\Delta_{k^{\prime}}^{2}\right)^{1 / 2}} .
$$

We assume the simple model for the interaction matrix

$$
V_{k k^{\prime}}=V \quad \text { if }\left|\mathcal{E}_{k}\right|<\omega_{D}, \quad V_{k k^{\prime}}=0 \text { otherwise. }
$$

We obtain for the energy gap:

$$
\Delta=\sqrt{\Lambda}=\frac{\omega_{D}}{\sinh [1 / V D]},
$$

where $D$ is the fermion density of states defined by the Fermi sphere for $N$ fermions by

$$
n_{f}=\int_{k_{0}}^{k_{f}} d^{3} k D(k)
$$

and $\omega_{D}(\hbar=1)$ is the Debye energy.

The physical density of states behaves for an expanding universe as

$$
D_{\text {phys }}\left(\omega_{k}\right) \sim a^{3}(t),
$$

so that as the universe inflates and $a(t) \rightarrow \infty$, we have $D(\omega) \rightarrow \infty$. However, we assume as a first approximation that $V D$ is constant and independent of $a(t)$.

We find that

$$
V_{k k^{\prime} q}=\frac{\left|M_{q}\right|^{2} \omega_{D}}{\left(\mathcal{E}_{k}-\mathcal{E}_{k-q}\right)^{2}-\omega_{D}^{2}} \sim \frac{m_{f}}{M_{f}}\left(\frac{N V_{k}}{\omega_{D}}\right)^{2} .
$$

Here, $m_{f}$ and $M_{f}$ denote the negatively charged condensate fermion mass and the positively charged fermion "ion" mass, respectively.

In the early universe there is an initial phase in which spacetime is flat (Minkowski) and the fermions do not interact gravitationally. This phase is unstable to gravitational interactions between fermions. There is a phase transition to an inflating de Sitter vacuum, in which $V D \sim 1$ corresponding to a strong coupling limit and

$$
\Delta_{i}=\sqrt{\Lambda} \sim \omega_{D}
$$

In this phase

$$
\Delta_{i} \sim \omega_{D} \sim M_{\mathrm{PL}}
$$

where $M_{\mathrm{PL}}$ is the Planck mass. As the universe expands exponentially, a weak coupling limit develops when

$$
V D \ll 1
$$


which leads to an exponential suppression

$$
\Delta_{f}=\sqrt{\Lambda}=2 \omega_{D} \exp \left(-\frac{1}{V D}\right)
$$

We see that the fermion condensate phase can generate enough inflation initially and then as the universe expands towards the present epoch produce an exponential suppression of the cosmological constant, leading to a zero or small value of $\Lambda$ in the present universe.

The condensation energy for the weak coupling limit $V D \ll 1$ is given by

$$
\mathcal{E}_{\text {cond }} \sim-2 \omega_{D}^{2} D \exp \left(-\frac{2}{V D}\right) \sim-\frac{1}{2} D \Lambda^{2} .
$$

Thus, as the universe expands from its initial inflationary period the number of fermions that is affected by the attractive gravitational and screened interaction is a small fraction of the total number of fermions in the universe. We note that $\exp [-2 /(V D)]$ has an essential singularity at $V=0$, which means that while the function and its derivatives vanish as $V \rightarrow+0$, they all become infinite as $V \rightarrow-0$. This means that we cannot calculate $\mathcal{E}_{\text {cond }}$ by using perturbation theory.

What about the fluctuations that cause ultraviolet divergences? We claim that these have to be subtracted out from the total vacuum energy and do not play a fundamental role in the calculation of the vacuum energy. This is the procedure used to calculate the Casimir vacuum energy inside, for example, a spherical ball with thin walls. The cosmological constant problem arises from physics in the lowenergy, infrared domain and the ultraviolet divergences will be removed by a future self-consistent non-perturbative quantum field theory and quantum gravity theory.

\section{Vacuum Instability in the de Sitter Phase}

Let us illustrate this mechanism with a toy model which effectively models the formation of "Cooper-pairs" in a relativistic context. Consider the following theory with an action describing massless fermions coupled to gravity 6 .

$$
\mathcal{L}=\sqrt{-g}\left[R+\sum_{k=1}^{N} \bar{\psi} D_{a} \gamma^{a} \psi+\sum_{k=1}^{N} \frac{G_{0}}{2 N}(\bar{\psi} \psi \bar{\psi} \psi)\right],
$$

where $D_{a}$ is the covariant derivative with respect to the local spin connection, $G_{0}$ is the "bare" gravitational coupling constant, $N$ is the number of fermion species, and the third term is a four-fermion interaction, which describes the relevant graviton interaction between pairs of fermions on the Fermi surface.

It is well-known that the physics described by (20) is equivalent to the following Lagrangian

$$
\mathcal{L}=\sqrt{-g}\left[R+\bar{\psi} D_{a} \gamma^{a} \psi+\bar{\psi} \phi \psi-\frac{N}{2 G_{0}} \phi^{2}\right],
$$

where $\phi=<\bar{\psi} \psi>$ is the condensate which forms from fermion-graviton interactions. 
As a simple first step, we will consider graviton exchange between pairs of fermions by expanding about Minkowski spacetime:

$$
g_{\mu \nu}=\eta_{\mu \nu}+h_{\mu \nu}+O\left(h^{2}\right)
$$

where $\eta_{\mu \nu}$ is the background Minkowski space metric: $\eta_{\mu \nu}=\operatorname{diag}(+1,-1,-1,-1)$. For a Dirac fermion, we obtain for a momentum cutoff $K_{c}$ in the weak curvature limit

$$
i \gamma^{\mu} p_{\mu}+\sqrt{\Lambda_{0}}+\Sigma\left(p, \sqrt{\Lambda}, G_{0}, K_{c}\right)=0
$$

for $i \gamma^{\mu} p_{\mu}+\sqrt{\Lambda}=0$.

We have for a zero bare cosmological constant, $\Lambda_{0}=0$ :

$$
\sqrt{\Lambda}=\Sigma .
$$

For gravity for the lowest-order loop we obtain

$$
\sqrt{\Lambda}=G_{0} \sqrt{\Lambda} F\left(\sqrt{\Lambda}, K_{c}\right),
$$

where $F\left(\sqrt{\Lambda}, K_{c}\right)$ is the result of the momentum integration of the Feynman fermion propagators and the cutoff is $K_{c}=M_{\mathrm{PL}}$, where $M_{\mathrm{PL}}$ is the Planck mass. This has two solutions: either $\sqrt{\Lambda}$ is zero or

$$
\frac{1}{G_{0}}=F\left(\sqrt{\Lambda}, K_{c}\right)
$$

The first solution is the trivial perturbative solution, while the second, nontrivial non-perturbative solution determines $\sqrt{\Lambda}$ in terms of the bare gravitational coupling constant $G_{0}$ and the cutoff $K_{c}$. The nontrivial solution corresponds to the superfluid condensate state which is the true vacuum state of the system, while the trivial solution corresponds to the normal (false) vacuum state i.e. not to the true vacuum state $^{\mathrm{a}}$.

The gap equation (26) asymptotically has an exponential dependence on the physical density of states. We have that in an FRW background filled with fermions the energy gap will take on the following form, $\Delta=\sqrt{\Lambda}$, separating the two phases. Physically this means that the gap corresponds to the binding energy necessary to form the condensate. The difference between the original vacuum energy and the final vacuum energy is the rest mass of the condensate.

\section{Conclusions}

We have shown that the vacuum energy in the early universe can become unstable as the attractive fermion-gravitational force and a screened attractive force between fermions produces condensates through a phase transition at a critical temperature $T<T_{c}$.

${ }^{a}$ A de Sitter space solution to the non-perturbative gap equation has been obtained for a large $N$ expansion by Inagaki et al. 7 . 
The non-perturbative phase transition generates a gap in the ground state or vacuum energy and we make the identification $\Delta \sim \sqrt{\Lambda}$. An initial phase of de Sitter inflation caused by a large vacuum energy (cosmological constant) is followed by an exponential suppression of the cosmological constant as the universe expands. We described this scenario by analogy with the BCS mechanism associated with the formation of Cooper pairs of fermions by means of the exchange of phonons, replacing the phonon exchange by graviton exchange.

The non-perturbative mechanism can explain how an initially large vacuum energy (cosmological constant) can be suppressed by a phase transition to a superfluid state of the early universe as the universe expands, leading to a "graceful" exit for inflation.

The non-relativistic model we have used to describe the scenario can be extended to a relativistic QFT model of the formation of a vacuum energy gap for fermion condensates in a de Sitter spacetime background.

This scenario explains why a naive perturbative calculation of the vacuum energy leads to a nonsensical answer. In contrast to the ground state or vacuum of QED or the standard model, the vacuum associated with gravity is unstable and the instability can only be described by non-perturbative physics.

\section{Acknowledgments}

This work was supported by the Natural Sciences and Engineering Research Council of Canada. I wish to thank my collaborator Stephon Alexander for stimulating and helpful discussions.

\section{References}

1. S. Weinberg, Rev. Mod. Phys. 61, 1 (1989).

2. N. Straumann, astro-ph/020333.

3. Stephon Alexander, Manasse Mbonje and John Moffat, hep-th/0406202

4. J. Bardeen, L. N. Cooper and J. R. Schrieffer, Phys. Rev. 108, 1175 (1957).

5. P. L. Taylor and O. Heinonen, A Quantum Approach to Condensed Matter Physics, Cambridge University Press, 2002.

6. Y. Nambu and G. Jona-Lasinio, Phys. Rev. 122, 345 (1961).

7. T. Inagaki, S. Mukaigawa and T. Muta, Phys. Rev. D52, 4267 (1995), hep-th/9505058 